\title{
Der ökonomische Wert der Währungsunion: eine positive Bilanz aus deutscher Sicht
}

\author{
Christian Dreger*
}

Die schnelle Transformation der Finanz- und Wirtschaftskrise in eine Schuldenkrise der öffentlichen Haushalte bedroht inzwischen die Stabilität im Euroraum. Nachdem die Krise zunächst auf Griechenland begrenzt war, haben die Verwerfungen auch Kernstaaten der Währungsunion erreicht. Obgleich sich nach dem erfolgreichen Schuldenschnitt für Griechenland die Lage zwischenzeitlich etwas beruhigt hat, kann sich die Krise schnell wieder verschärfen. $\mathrm{Zu}$ den steigenden Ansteckungsgefahren haben auch die wenig überzeugenden Antworten der Politik beigetragen. Das derzeitige wirtschaftspolitische Krisenmanagement kann eine Stagflation, also eine Entwicklung mit geringen Wachstumsraten bei relativ hoher Inflation verfestigen. Die Arbeitslosigkeit ist in vielen Staaten deutlich gestiegen, insbesondere bei jüngeren Arbeitnehmern, die in den Arbeitsmarkt eintreten möchten. Die Finanzmärkte sind noch immer volatil. Die Bereitschaft zur Kreditgewährung hat sich am Interbankenmarkt weiter verschlechtert. Der Geldschöpfungsmultiplikator, definiert als das Verhältnis der inflationsrelevanten Geldmenge M3 zur Geldbasis, liegt nach Angaben der Europäischen Zentralbank noch um etwa 20 Prozent unter dem Vorkrisenniveau. Sofern sich die Verhältnisse wieder normalisieren, ist mit einer starken Ausweitung der Liquidität zu rechnen, die mittel- und langfristig einen erheblichen Preisdruck erzeugen dürfte.

Die Herausforderung für die wirtschaftspolitischen Entscheidungsträger besteht darin, die konjunkturelle Erholung trotz der Schuldenkrise zu fördern und gleichzeitig die Konsolidierung der öffentlichen Haushalte voranzutreiben. Dabei ist Ziel der Europa 2020-Strategie, ${ }^{1}$ ein innovationsgetriebenes Wachstum durch ein stabilitätsorientiertes makroökonomisches Management abzusichern. Derzeit ist noch unklar, ob dies tatsächlich gelingt. So ist mancherorts bereits das Ende der Gemeinschaftswährung prophezeit worden. Einige Ökonomen haben Griechenland den Austritt aus der Währungsunion und eine Rückkehr zur Drachme empfohlen, nicht zuletzt, um die Bedeutung des Euro im internationalen Währungssystem wieder zu stärken. ${ }^{2}$

In der Diskussion ist für die politischen Entscheidungsträger und die Bürger von Interesse, ob die Kosten oder die Nutzen der Währungsunion überwiegen. Dieser Artikel beleuchtet die Argumente durch die deutsche ,Brille‘. Im ersten Schritt wird die makroökonomische Entwicklung vor und nach Einführung der Währungsunion aufgezeigt. Der Schwerpunkt liegt auf Wachstum und Inflation. Im Anschluss daran werden die mikro- und makroökonomischen Effekte der Währungsunion diskutiert. Deutschland hatte in der ersten Phase der Währungsunion als Niedriginflationsland spezifische Nachteile. Dagegen überwiegen in den letzten Jahren die Vorteile, die sich nicht zuletzt aufgrund der Strukturreformen auf den Arbeitsmärkten eingestellt haben. Im dritten Schritt wird schließlich eine Wertung der Ergebnisse vorgenommen.

* Prof. Dr. Christian Dreger, Europa-Universität Viadrina Frankfurt an der Oder und Deutsches Institut für Wirtschaftsforschung (DIW), Berlin.

1 Europäische Kommission: Mitteilung der Kommission. Europa 2020 Eine Strategie für intelligentes, nachhaltiges und integratives Wachstum, KOM (2010) 2020.

2 Siehe beispielsweise Hans-Werner Sinn: Wir sitzen in der Falle, in: Frankfurter Allgemeine Zeitung, 18.2.2012. 


\section{Entwicklung von realwirtschaftlichem Wachstum und Inflation}

Legt man die bisherigen makroökonomischen Erfahrungen zugrunde, ist die Währungsunion nicht ohne Weiteres als Erfolgsgeschichte zu werten. So ist das mittlere Wachstum des realen Bruttoinlandsprodukts in den großen Mitgliedstaaten seit den 1980er Jahren im Trend gesunken (siehe Abbildung 1). Das gilt uneingeschränkt für Italien und Frankreich. In Deutschland zeigt sich seit einiger Zeit eine Umkehr, deren Nachhaltigkeit jedoch noch abzuwarten bleibt. Das Wachstum in Staaten der Peripherie wie Spanien und Portugal hat dagegen bis zur Finanzkrise deutlich angezogen. Das Wachstum in der gesamten Währungsunion war in den meisten Jahren relativ niedrig. Damit haben sich die hochfliegenden Wachstumserwartungen, die an den Übergang zur Gemeinschaftswährung gekoppelt waren, zumindest bisher nicht erfüllt.

Bei der Inflationsentwicklung sind hingegen Erfolge zu verzeichnen (siehe Abbildung 2). Vom nachlassenden Preisdruck haben vor allem Staaten mit vorher hohen Inflationsraten wie Italien und Spanien profitiert. Auch in Deutschland hat der allgemeine Preisauftrieb nachgelassen, wenngleich nur marginal, weil man hierzulande bereits vor der Währungsunion niedrige Inflationsraten aufweisen konnte. Anstelle einer jahresdurchschnittlichen Teuerung von 2,2 Prozent liegt die Inflation im langjährigen Mittel nun bei 1,6 Prozent. Allerdings ist gegenwärtig wieder ein stärkeres Anziehen der Preise zu konstatieren, das auf anziehende Lebensmittel- und Rohstoffpreise, aber auch auf die günstige konjunkturelle Entwicklung zurückzuführen ist.

\section{Abbildung 1: Makroökonomische Entwicklung vor und nach Einführung der Wäh- rungsunion: Wachstum des realen Bruttoinlandsprodukts}

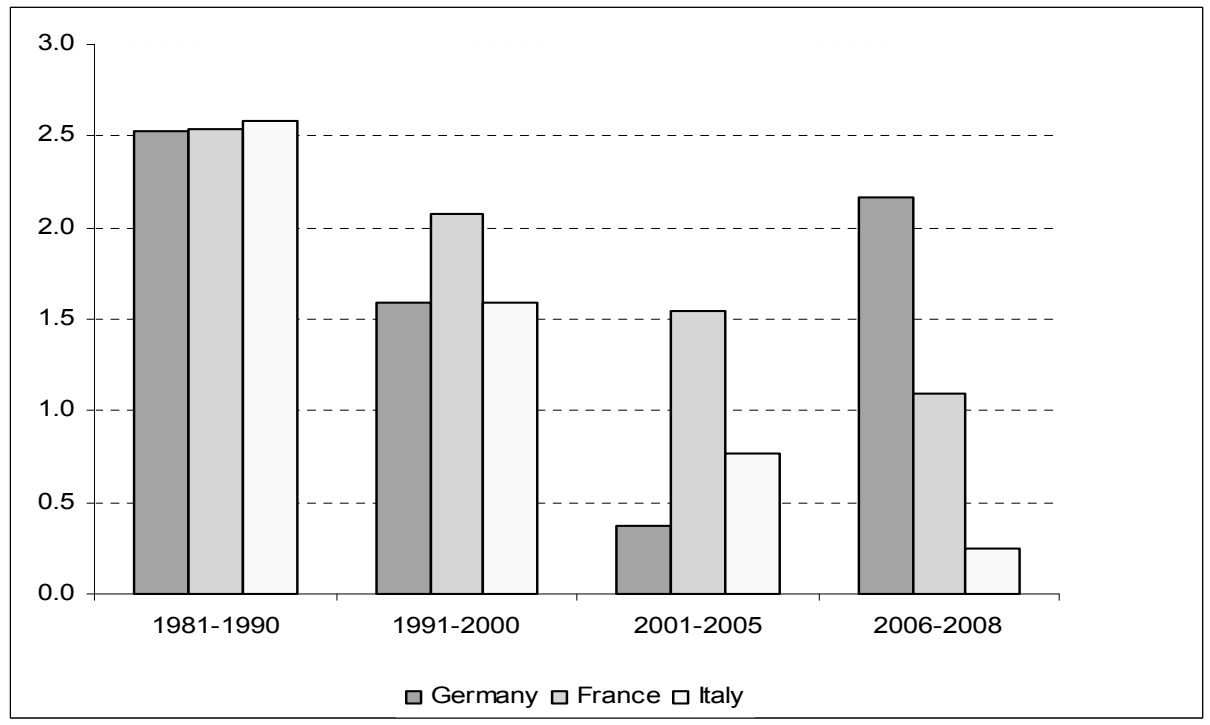

Quelle: Eurostat. 


\section{Abbildung 2: Makroökonomische Entwicklung vor und nach Einführung der Wäh- rungsunion: Inflation der Konsumentenpreise}

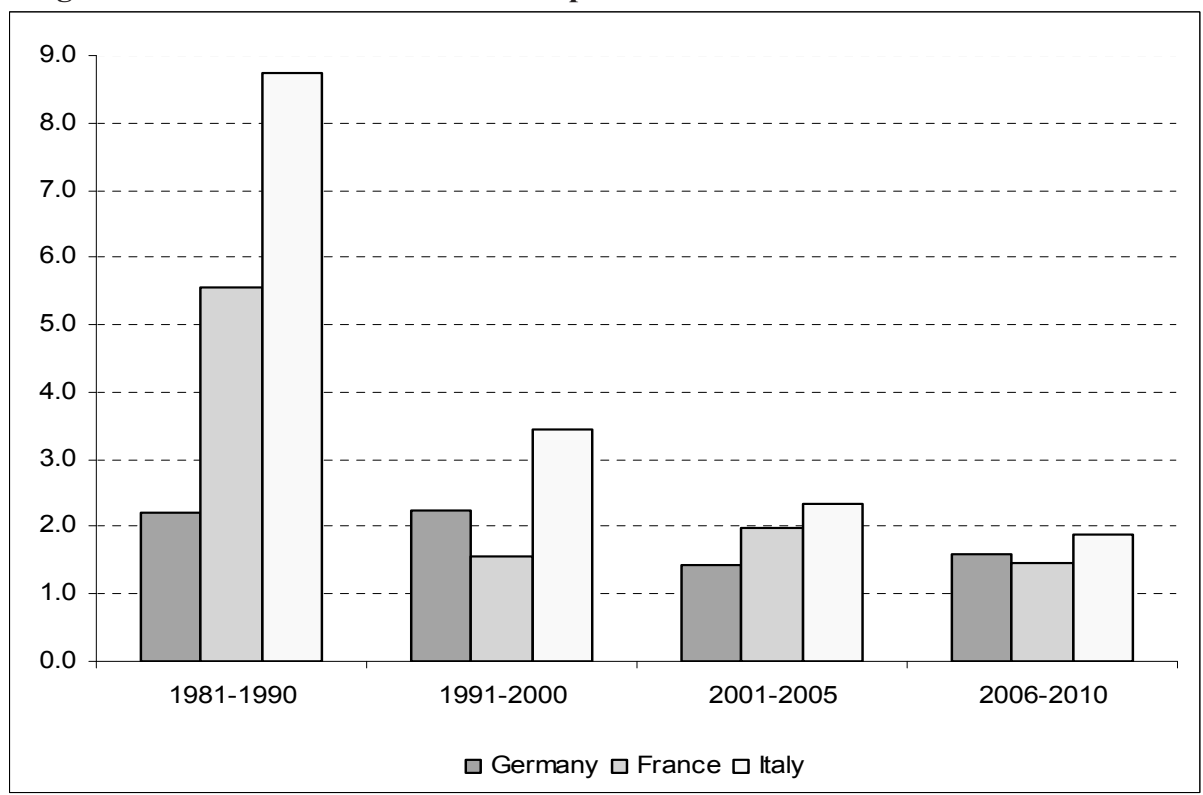

Quelle: Eurostat.

Die unterschiedlichen Inflationsraten in der Währungsunion haben zu erheblichen Divergenzen in den Realzinsen beigetragen, die die Entwicklung der Konsum- und Investitionsnachfrage beeinflussen. So ist es mit dem Beitritt der Staaten zur Währungsunion zu einer raschen Konvergenz der Nominalzinssätze auch am langen Ende des Marktes gekommen. Damit war trotz der einheitlichen Geldpolitik keineswegs zu rechnen. Internationale Investoren haben im Vorgriff auf ein stärkeres Wachstum in den Peripheriestaaten nicht mehr zwischen den Staatsanleihen unterschiedlicher Länder differenziert. Zudem sind vormals bestehende Risiken einer Abwertung der jeweiligen Landeswährung mit dem Übergang zum Euro entfallen. Daneben ist die Europäische Zentralbank als Garant stabiler Preise in das Anlagekalkül einbezogen worden. Damit konnte sich beispielsweise Griechenland bis zur Finanzkrise an den Kapitalmärkten zu ähnlichen Zinssätzen wie Deutschland verschulden. Aufgrund seiner niedrigeren Inflationsrate hatte Deutschland im Vergleich zu seinen Partnerstaaten höhere Realzinssätze und damit einen Kostennachteil zu verkraften, der die Entwicklung von Konsum- und Investitionsnachfrage behindert hat. Dieser Mechanismus legt ein destabilisierendes Element der Währungsunion offen: Hat ein Staat höhere Realzinsen, dämpft dies die Nachfrage, sodass der Preisdruck im betreffenden Staat weiter abnimmt und die ursprüngliche Divergenz der Realzinssätze noch vergrößert. Die Kostennachteile Deutschlands sind über Jahre hinweg durch eine zurückhaltende Lohnpolitik abgebaut worden. Auch die Strukturreformen an den Produkt- und Arbeitsmärkten haben dazu beigetragen, die internationale Wettbewerbsfähigkeit zu verbessern.

Die skizzierte Entwicklung lässt sich keineswegs allein der Währungsunion zuschreiben. Tatsächlich sind andere Ereignisse wie die Deutsche Wiedervereinigung zentraler, um den hiesigen Befund zu erklären. Darüber hinaus ist Deutschland wegen seiner Exportorientie- 
rung in hohem Maße dem internationalen Wettbewerb ausgesetzt, der wegen der fortschreitenden Globalisierung der Märkte noch weiter zunehmen wird. Der Anpassungsdruck hat sich allerdings durch den Prozess der europäischen Integration verschärft. Dabei spielt neben der EU-Osterweiterung die Währungsunion eine entscheidende Rolle. Auch der Rückgang der Inflation ist keineswegs allein einer erfolgreichen Geldpolitik der Europäischen Zentralbank zuzuschreiben. So haben die Währungshüter ihr Ziel einer Geldmengensteigerung von 4 bis 5 Prozent pro Jahr oft drastisch verfehlt. In vielen Jahren ist die Liquidität mit Raten um 10 Prozent deutlich stärker ausgedehnt worden. Gleichwohl hat sich die Inflation rückläufig entwickelt. Dahinter steht neben einem intensiveren Wettbewerb die stärkere Aufspaltung von Produktionsketten, die mit der Integration der Schwellenstaaten in die Weltwirtschaft einhergeht. So wurden arbeitsintensive Produktionen häufig in Niedriglohnländer ausgelagert, um Lohnkosten zu sparen. Die Koordinierung von Produktionsstufen hat sich mit der Einführung neuer Kommunikationstechnologien wie dem Internet deutlich verbessert. Auch die trendmäßige Aufwertung des Euro hat dazu beigetragen, dass der Druck von den internationalen Rohstoff- und Lebensmittelmärkten weniger stark auf heimische Preisentwicklung und Kaufkraft der privaten Haushalte durchschlägt.

\section{Wachstumseffekte der Währungsunion}

Die Einführung der Gemeinschaftswährung hat unterschiedliche Wachstumseffekte ausgelöst. Während die statischen Wirkungen an den Zeitraum der Anpassung an die neuen Rahmenbedingungen gebunden sind und nur einen einmaligen Wachstumsschub auslösen können, sind die dynamischen Wirkungen geeignet, ein dauerhaft höheres Wachstum zu erklären. So führen die Abnahme von Transaktionskosten und der Wegfall von Währungsrisiken nur zu kurzfristigen Wachstumsimpulsen. Dagegen dürfte der steigende Wettbewerb im Euroraum das Innovationsgeschehen auch längerfristig beflügeln, sodass dauerhaft ein höherer Wachstumspfad erreichbar ist. Wie die tatsächliche Entwicklung zeigt, sind die Steigerungen der Produktion in den meisten Jahren nur moderat ausgefallen, ein lang anhaltender Wachstumsschub ist bislang ausgeblieben. Der Euroraum zählt zumindest nicht zu den am schnellsten wachsenden Regionen der Weltwirtschaft. Dazu hat insbesondere das recht schwache Wachstum in den großen Staaten beigetragen.

Allerdings hat der Euro den Außenhandel stimuliert. Vor der Einführung der Gemeinschaftswährung hatten starke Ausschläge von Wechselkursen den internationalen Handel behindert. Viele Unternehmen, die vorher keine Güter in andere Mitgliedstaaten der Währungsunion ausführten, sind aufgrund der nun größeren Preistransparenz, der geringeren Transaktionskosten und dem Wegfall des Wechselkursrisikos zu Exporteuren geworden.

Auch die Lohnpolitik kann losgelöst von Schwankungen der nominalen Wechselkurse agieren. Vor der Einführung des Euro hatte man in Deutschland das Problem, dass die DMark beständig aufgewertet wurde, während die Währungen der südeuropäischen Staaten aufgrund höherer Inflationsraten immer wieder abgewertet wurden. Damit wurden deutsche Kostenvorteile wieder zunichte gemacht, was höhere Lohnabschlüsse erschwerte.

Vom Wegfall der Währungsrisiken profitieren vor allem exportorientierte Staaten wie Deutschland. Immerhin nehmen die Partnerstaaten im Euroraum rund 45 Prozent der deutschen Ausfuhren ab. Die höchsten Wachstumsraten haben sich allerdings im Handel mit den Staaten Mittel- und Osteuropas ergeben, die nicht der Währungsunion angehören. Daneben konnten in den letzten Jahren die Ausfuhren in den südostasiatischen Wirtschaftsraum deutlich gesteigert werden. 
Die Einführung der Gemeinschaftswährung bringt auch für die Verbraucher erhebliche Vorteile. So können sich Urlauber in den Eurostaaten die Kosten für einen Währungstausch und die Absicherung von Wechselkursrisiken sparen. Dies gilt freilich vor allem für die Verbraucher, die aus den vorher abwertungsgefährdeten Staaten stammen. Darüber hinaus trägt der höhere Wettbewerb auf den Gütermärkten dazu bei, die Inflation im Zaum zu halten und so die Kaufkraft der Verbraucher zu stabilisieren. Allerdings sind diese Vorteile nicht immer als solche erkannt worden. Seit der Einführung der Währungsunion haben sich insbesondere die Preise für Lebensmittel erhöht. Diese Güter werden täglich gekauft, sodass Preisbewegungen in diesem Segment besonders stark wahrgenommen werden. Der Anstieg der Lebensmittelpreise ist zwar nicht dem Euro anzulasten, führt aber dazu, dass die gefühlte Teuerung die tatsächliche Preissteigerung übertrifft. Dies schwächt die Reputation der Gemeinschaftswährung in der Bevölkerung.

Die Währungsunion bietet auch Schutz gegen externe Schocks. In der Finanzkrise wären ohne den Euro vor allem die Währungen der südeuropäischen Staaten massiv unter Druck geraten, möglicherweise hätte es sogar Währungskrisen gegeben. Massive Abwertungen in den südeuropäischen Staaten und eine drastische Aufwertung der D-Mark, die dadurch befördert worden wäre, dass noch mehr zusätzliches Kapital in die als sicher geltenden deutschen Staatsanleihen geflossen wäre, hätten Einbrüche im Export und gravierende Arbeitsplatzverluste nach sich gezogen. Dies gilt auch, wenn berücksichtigt wird, dass nur ein Teil der deutschen Exporte hoch preissensitiv ist. So bestehen die Ausfuhren nach Angaben der Außenhandelsstatistik zu 40 Prozent aus Investitionsgütern, die weniger anfällig für Wechselkursschwankungen sind. Käme es bei einem Auseinanderfallen des Euroraums nur zu moderaten Aufwertungen der D-Mark, dürften sich die negativen Auswirkungen auf die Exportaktivitäten in Grenzen halten. Realistischer ist, dass sich die preisliche Wettbewerbsfähigkeit deutscher Produkte nachhaltig verschlechtern und die Wachstumsperspektiven für einige Jahre erheblich reduzieren dürften.

Darüber hinaus kann die einheitliche Geldpolitik nicht nur zu höherer Stabilität beitragen. Sie führt auch zu Kosten, weil die Geldpolitik nicht auf die speziellen Bedürfnisse einzelner Staaten zugeschnitten ist. So unterscheidet sich die konjunkturelle Entwicklung zwischen den Mitgliedstaaten im Euroraum erheblich. Die gleiche Geldpolitik, die in einem Staat expansiv wirkt, kann aus Sicht des anderen Staates zu restriktiv ausgerichtet sein. Zum Beispiel ist die aktuelle Geldpolitik der Europäischen Zentralbank aus deutscher Sicht eher zu locker, während vor allem die Defizitstaaten eine stärkere Stimulierung der Konjunktur begrüßen würden, indem die Zentralbank die Inflationserwartungen schürt und so indirekt die Realzinssätze weiter senkt.

Fiskalpolitische Kosten entstehen unter anderem aus der Bewältigung der Schuldenkrise, die derzeit noch keineswegs ausgestanden ist. Die Schulden und Haushaltsprobleme eines einzelnen Staates sind nicht mehr länger dessen rein interne Angelegenheit. Die Kosten sind noch nicht in voller Höhe absehbar und umfassen zum einen den Schuldenerlass für Griechenland und eventuell weitere Schuldenerlasse für die notleidenden Staaten. Zum anderen ist die Finanzierung konjunkturstützender Maßnahmen in den Defizitstaaten erforderlich, um das dortige Wachstum anzuschieben. Andernfalls droht ein Absturz der wirtschaftlichen Aktivität wie derzeit in Griechenland, der die politische Akzeptanz des Konsolidierungsprozesses infrage stellt. Und schließlich würde eine Vereinheitlichung der Fiskalpolitik im Euroraum nationale Anpassungskapazitäten abbauen und auf diese Weise den Weg in eine Transferunion ebnen. 


\section{Bewertung}

Insgesamt dürften aus deutscher Sicht in den ersten Jahren die Nachteile der Währungsunion überwogen haben. Die Divergenz der Realzinssätze hat zu einer Umverteilung des Wirtschaftswachstums hin zu den Peripheriestaaten des Euroraums geführt. Deutschland hat jedoch seine Wettbewerbsfähigkeit durch längere Lohnzurückhaltung und strukturelle Reformen auf den Produkt- und Arbeitsmärkten gesteigert, sodass sich dieser Prozess in den letzten Jahren umgekehrt hat. Die Rendite des Anpassungsprozesses zeigt sich nicht zuletzt in einer relativ robusten konjunkturellen Entwicklung. Aufgrund der hohen internationalen Wettbewerbsfähigkeit der Produkte konnte der durch die Finanzkrise verursachte Einbruch schnell überwunden werden. Zudem profitiert Deutschland derzeit von seinem Status als sicherer ,Hafen ‘ für internationale Kapitalanleger, was das Niveau der langfristigen Zinsen senkt und für eine zusätzliche konjunkturelle Stimulierung sorgt. Die Partnerstaaten haben dagegen Risikoprämien zu zahlen, die die Refinanzierungskosten erhöhen und fiskalische Spielräume eng begrenzen. Bei einem Auseinanderbrechen des Euroraums bleiben wesentliche Integrationsfortschritte wie die Schaffung eines einheitlichen Binnenmarktes zwar erhalten. Dennoch sind gravierende Nachteile zu erwarten, die sich aus einer drastischen Aufwertung bei einer Wiedereinführung der D-Mark einstellen würden. Dabei ist entscheidend, wie sich die Ansteckungseffekte tatsächlich gestalten. Kommt es nur zu einem Austritt Griechenlands, dürften die negativen Folgen alles in allem verkraftbar sein. Allerdings sollten die wirtschaftspolitischen Entscheidungsträger nicht das psychologische Moment unterschätzen, das an den Kapitalmärkten mit einem solchen Schritt verbunden wäre.

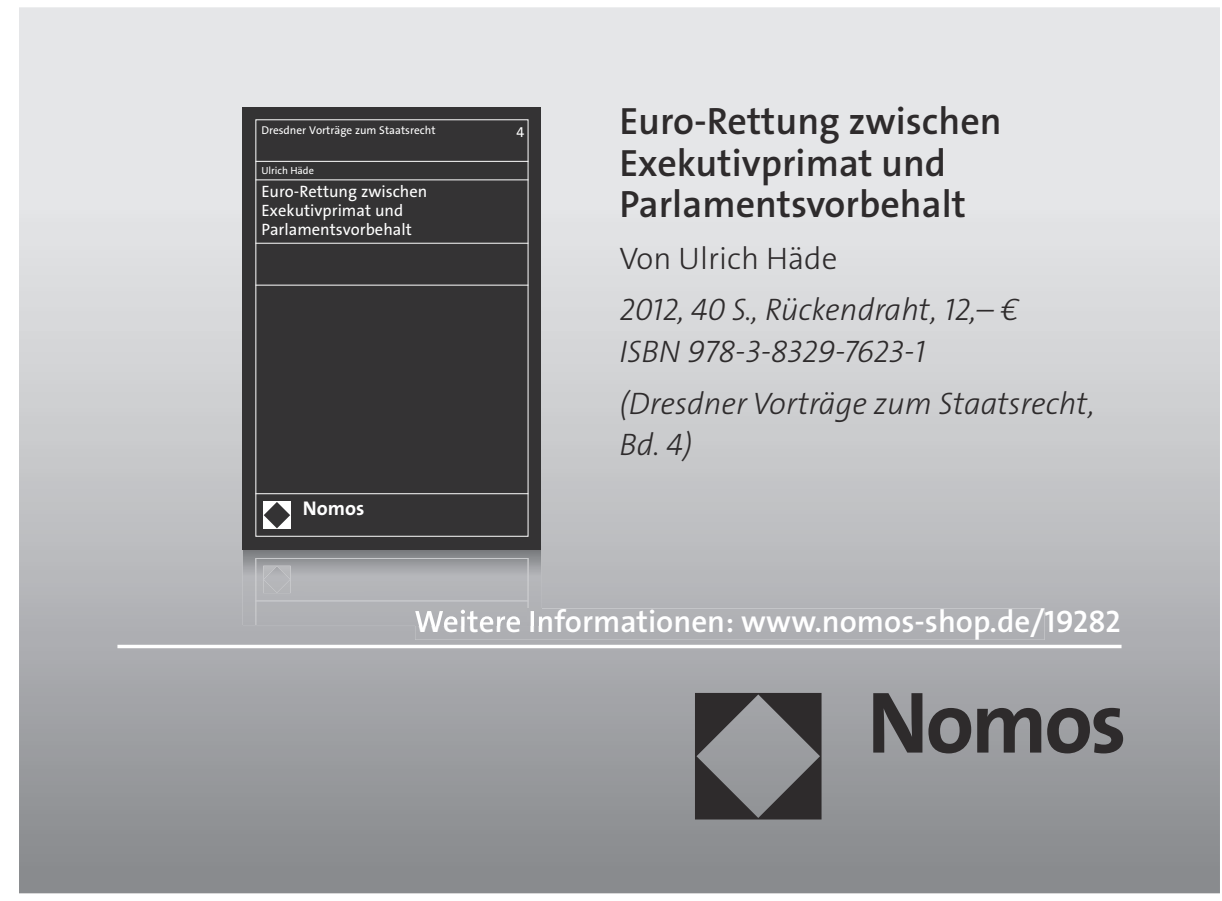

